\title{
COMPARISON BETWEEN IMAGE-FREE ROBOTIC ASSISTED AND CONVENTIONAL TOTAL KNEE ARTHROPLASTY: POSTOPERATIVE CT ASSESSMENT OF ALIGNMENT
}

\author{
Saradej Khuangsirikul, Siripat Popuk, Danai Heebthamai, Ong-art Phruetthiphat, \\ Thanainit Chotanaphuti
}

Department of Orthopedic Surgery, Phramongkutklao College of Medicine, Bangkok, Thailand

\begin{abstract}
Background: Navio Surgical System, a newer-generation robotic technology, is an image-free roboticassisted total knee arthroplasty (TKA) offering several advantages over older versions, including CT scan, the potential to dynamically assess soft tissue over a range of motion and the ability to use haptic control in bone preparation.

Objective: The study aimed to compare the accuracy of component alignment between image-free robotic-assisted and conventional TKA.

Methods: Forty patients were randomly assigned to two groups, 20 image-free robotic-assisted and 20 conventional TKA. The primary outcome was prosthetic alignment including mechanical axis alignment, epicondylar axis alignment and posterior tibial slope. The secondary outcomes included postoperative blood loss and operative time.

Results: Significant difference were found in the postoperative mechanical axis between the image-free robotic group and the conventional group $\left(1.15^{\circ} \pm 1^{\circ}\right.$ vs. $1.88^{\circ} \pm 1.19^{\circ}$ deviated from neutral mechanical alignment, $p=0.043$ ). There was significant difference in femoral rotational alignment between groups $\left(1.00^{\circ} \pm 0.75^{\circ}\right.$ vs. $2.33^{\circ} \pm 0.96^{\circ}$ deviated from the epicondylar axis, $\left.p<0.001\right)$. The mean posterior tibial slope did not significantly differ $\left(3.89^{\circ} \pm 1.66^{\circ}\right.$ vs. $\left.4.12^{\circ} \pm 1.37^{\circ}, p=0.639\right)$. The operative time in the image-free robotic group was significantly longer than that of the conventional group $(102.80 \pm 11.18$ min vs. $62.90 \pm 3.28 \mathrm{~min}, p<0.001)$. Total blood loss in the image-free robotic group was significantly higher than conventional group $(2.24 \pm 0.49 \mathrm{~g} / \mathrm{dl}$ vs. $1.64 \pm 0.68 \mathrm{~g} / \mathrm{dl}, p=0.001)$.

Conclusion: Image-free robotic-assisted TKA constituted a surgical procedure which could provide better accuracy in prosthetic alignment in both mechanical axis and rotational axis compared with conventional TKA. However, the image-free robotic assisted TKA involved higher blood loss and longer operative time.
\end{abstract}

Keywords: Image-free robotic-assisted TKA, Robotic knee surgery, NAVIO, Mechanical axis alignment, Rotational axis alignment

J Southeast Asian Med Res 2020; 4(1):16-23

http://www.jseamed.org

Correspondence to:

Khuangsirikul S., Department of Orthopedic Surgery, Phramongkutklao College of Medicine, Bangkok, Thailand

E-mail : Ksaradej@yahoo.com

Received: 14 February 2020

Revised: 15 April 2020

Accepted: 12 May 2020 


\section{Introduction}

Total knee arthroplasty (TKA) is a standard treatment for pain relief and restoration of joint function in the advanced arthritic knee. Studies for conventional TKA have revealed some mechanical axis outliers ${ }^{(1-4)}$ affecting patient satisfaction and implant longevity; ${ }^{(5-8)}$ the mechanical axis exceeding $\pm 3^{\circ}$ varus/valgus deviation has been reported with abnormal force, aseptic loosening and poor long-term outcomes. ${ }^{(9-10)}$ Femoral component rotational malalignment has been associated with anterior knee pain, patella-femoral maltracking and ligament balancing problems. ${ }^{(1-13)}$ To improve the accuracy of component alignment, computer-assisted and robotic systems have been developed for TKA. Robotic-assisted TKA increases the accuracy of component position ${ }^{(14-16)}$ and has reported only 0 to $2 \%$ of mechanical axis outliers. ${ }^{(14,16-20)}$ However, the previous generation of robotic systems were costlier and showed nonsignificant differences in clinical outcomes compared with conventional techniques. $^{(16,18,20-22)}$ Newer-generation robotic technology (Navio Surgical System) produces an image-free robotic-assisted TKA offering several advantages over older versions including CT scan, the potential to dynamically assess soft tissue over a range of motion and the ability to use haptic control in bone preparation. This novel technology has been used in unicompartmental knee arthroplasty (UKA) for several years and demonstrated superior outcomes over conventional techniques. ${ }^{(23)}$ Recently, it has been approved by the US Food and Drug Administration to use in TKA. No previous clinical study of this technology used for TKA has been conducted, so a well-designed clinical trial is required to investigate its efficacy. The study aimed to compare the accuracy of component alignment between image-free robotic -assisted (Navio Surgical System) and conventional TKA.

\section{Methods}

Primary osteo-arthritis knee patients undergoing total knee arthroplasty at Phramongkutklao Hospital from August 2018 to June 2019 were recruited to the study. Forty patients were previously prospectively randomized in two groups (20 image-free robotic-assisted and 20 conventional TKAs). Patients with an instrument extended into the knee, metal device that could cause CT scatter, deformity greater than 15 degrees of varus, valgus or flexion contracture, previous ipsilateral distal femoral or high tibial osteotomies, inflammatory arthritis, ankylosis of the hip joint on the side to be treated or previous patellectomy were excluded from the study. All patients were assigned to one of the two groups (1:1), group 1 was treated with image-free robotic-assisted TKA using the Navio Surgical System (Smith $\&$ Nephew Inc.), while group 2 was treated using conventional manual implantation. In both groups, we used the standard medial parapatellar approach and the same prosthesis (legion posterior stabilized knee, Smith \& Nephew, Memphis, TN, USA). The operation was performed by a single surgeon experienced in knee replacement surgery over 25 years.

\section{Surgical technique}

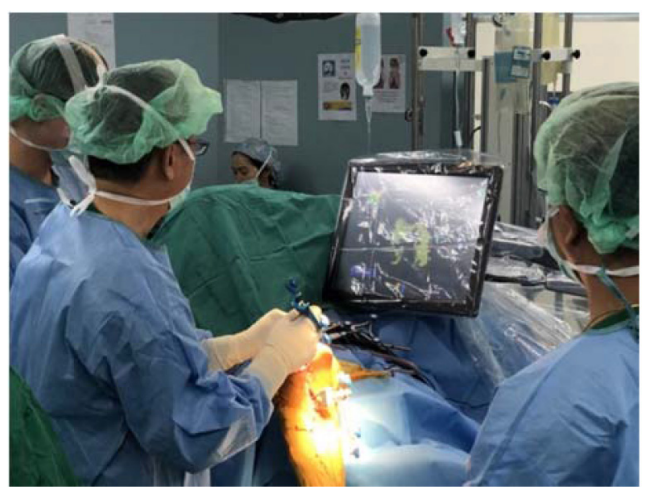

Image 1. 3D-model of the patient's cartilage and bone was captured using direct surface mapping by surgical probe, eliminating the need of pre-operative CT scan.

The Navio Surgical System was used in the image-free robotic assisted surgery group. Planning of implant position and bone resection were determined intra-operatively without need of pre-operative CT scan. After tracker fixation and landmark registration, range of motion and laxity mapping were performed. Anatomy of the femoral condyle and tibial plateau were mapped by painting the surfaces with an optical probe. A virtual model of the knee was thus created as shown in Image 1. Planning for component size, position, alignment and amount of bone removal were determined intra-operatively by the surgeon. Degenerative bone and cartilage were removed using the handheld sculptor as shown in Image 2. The system continuously tracked the position of the patient's lower limb and the 


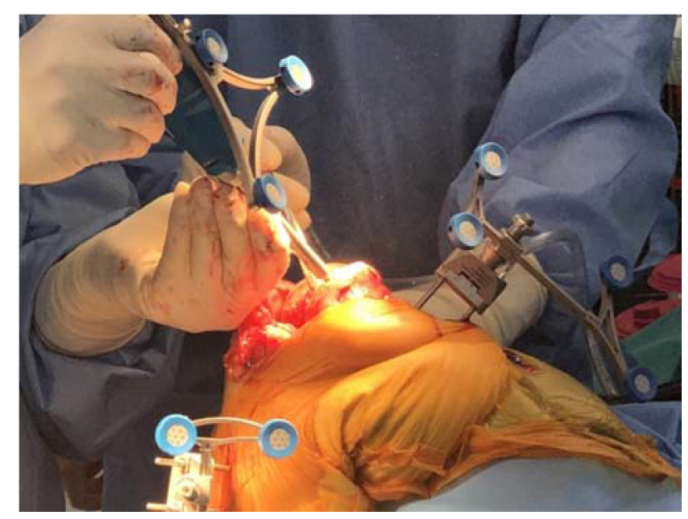

Image 2. NAVIO ${ }^{\mathrm{TM}}$ handheld burring technology removed only the bone determined by the surgeon's plan. Bone removal was seen on the NAVIO screen in real-time allowing the surgeon to continuously assess patient anatomy.

progress of bone resection using a navigation system camera.

For the conventional group, we performed the proximal tibial cut with an extramedullary guide, perpendicular to the mechanical axis and $3^{\circ}$ posterior slope. Then an intramedullary guide was used with $5^{\circ}$ valgus resection cut of the distal femur and external rotation was set at $3^{\circ}-5^{\circ}$ related to the posterior condyle of the femur (combined measurement and gap techniques). The patella was resurfaced in both groups. All patients received tranexamic acid $10 \mathrm{mg} / \mathrm{kg}$, one dose after start anesthesia and one dose before skin closure with no suction drain used in all cases. The operative times were also recorded. We also collected total blood loss calculated using hemoglobin change between pre-operative period and postoperative day 4 . At 6 weeks postoperative, all patients received a weight bearing radiograph of the hip-knee-ankle and CT scan of the knee.

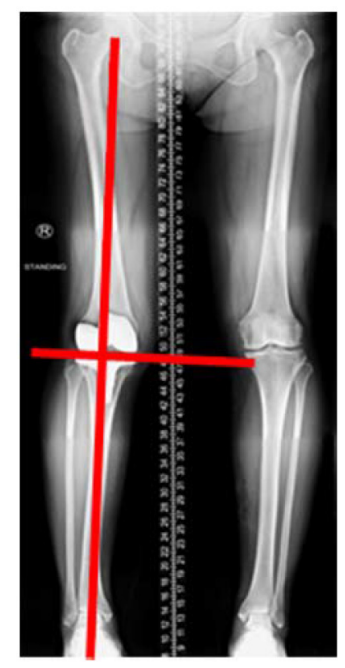

Image 3. Postoperative weight bearing radiograph was used for mechanical axis alignment measurement.
The results were evaluated by two adult reconstruction hip \& knee fellows unassociated with the treatment. Inter-observer reliability was analyzed using interclass correlation coefficient. The measurement used to determine component coronal alignment was the mechanical axis from the center of the femoral head to the center of the talus (Image 3). The accepted normal range of the mechanical axis did not exceed \pm 3 degrees of varus/valgus deviation. For the rotational alignment, we used the epicondylar axis, line connecting the medial epicondylar sulcus and the lateral epicondylar prominence, based on the technique described by Berger et al. ${ }^{(24-26)}$ (Image 4) The posterior tibial slope as also assessed. The chi-square test, independent t-test, Mann-Whitney U test and Fisher's exact test were used to compare baseline characteristics between groups. The analysis was performed using SPSS Software, Version 24. This study was approved by the Institutional Review Board of the Royal Thai Army Medical Department and all patients provided written informed consent.

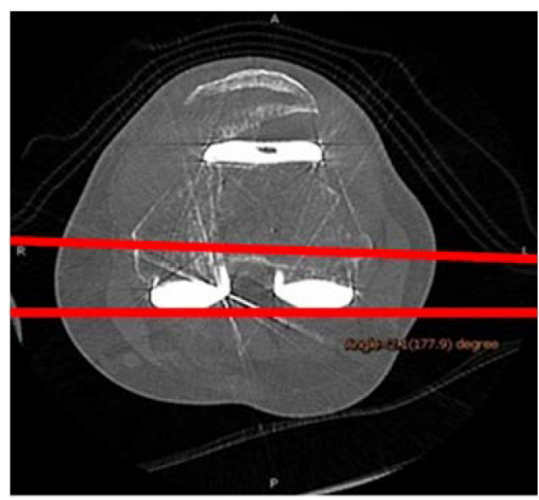

Image 4. Postoperative CT scan used for rotational axis alignment measurement. 
Table 1. Demographic characteristics of enrolled participants

\begin{tabular}{|c|c|c|c|}
\hline & $\begin{array}{l}\text { Robotic gr. } \\
\mathrm{N}=20\end{array}$ & $\begin{array}{l}\text { Conventional gr. } \\
\qquad \mathrm{N}=20\end{array}$ & $p$-value \\
\hline Age & $67.75 \pm 8.69$ & $70.95 \pm 5.74$ & 0.178 \\
\hline Sex & & & 1.000 \\
\hline Male & $2(10.00 \%)$ & $3(15.00 \%)$ & \\
\hline Firale & $18(90.00 \%)$ & $17(85.00 \%)$ & \\
\hline Female & & & \\
\hline Body mass index $\left(\mathrm{kg} / \mathrm{m}^{2}\right)$ & $28.8 \pm 4.22$ & $26.61 \pm 2.92$ & 0.054 \\
\hline
\end{tabular}

Table 2. Comparative results of component alignment, operative time and hemoglobin change

\begin{tabular}{|c|c|c|c|c|c|}
\hline & \multicolumn{2}{|c|}{ Robotic group } & \multicolumn{2}{|c|}{ Conventional group } & \multirow{2}{*}{$p$-value } \\
\hline & Mean \pm SD & Median (min-max) & Mean \pm SD & $\begin{array}{c}\text { Median } \\
(\text { min-max })\end{array}$ & \\
\hline Mechanical & $1.15^{\circ} \pm 1$ & $0.8^{\circ}\left(-1.2^{\circ}-4.7^{\circ}\right)$ & $1.88^{\circ} \pm 1.19$ & $-0.95^{\circ}$ & $0.043^{*}$ \\
\hline axis & & & & $\left(-4.2^{\circ}-2.8^{\circ}\right)$ & \\
\hline Rotational axis & $1.00^{\circ} \pm 0.75$ & $0.70^{\circ}\left(-1.6^{\circ}-3.1^{\circ}\right)$ & $2.33^{\circ} \pm 0.96$ & $\begin{array}{c}2.15^{\circ} \\
\left(-2.8^{\circ}-4.9^{\circ}\right)\end{array}$ & $<0.001^{*}$ \\
\hline Posterior slope & $3.89^{\circ} \pm 1.66$ & $3.5^{\circ}(1.2-7)$ & $4.12^{\circ} \pm 1.37$ & $\begin{array}{c}4^{\circ} \\
\left(2^{\circ}-7^{\circ}\right)\end{array}$ & 0.639 \\
\hline $\begin{array}{l}\text { Operative time } \\
(\mathrm{min})\end{array}$ & $102.80 \pm 11.18$ & $101(81-126)$ & $62.90 \pm 3.28$ & $\begin{array}{c}62.5 \\
(58-69)\end{array}$ & $<0.001^{*}$ \\
\hline $\begin{array}{l}\text { Hemoglobin } \\
\text { change }(\mathrm{g} / \mathrm{dl})\end{array}$ & $2.24 \pm 0.49$ & $2.3(1.6-3.5)$ & $1.64 \pm 0.68$ & $\begin{array}{c}1.7 \\
(0.5-3.3) \\
\end{array}$ & $<0.001 *$ \\
\hline
\end{tabular}

Mechanical axis: Negative values stand for varus; positive values stand for valgus.

Rotational alignment: Negative values stand for internal rotation; positive values stand for external rotation.

Posterior slope: Negative values stand for anterior slope; positive values stand for posterior slope.

* Statistically significant difference

\section{Results}

No significant difference was found between groups regarding mean age of patients, sex and BMI as shown in Table 1.

Significant differences were found in the postoperative mechanical axis between the image-free robotic group [mean $1.15^{\circ} \pm 1^{\circ}$ deviated from the neutral mechanical alignment (range, varus $1.2^{\circ}$ to valgus $4.7^{\circ}$ )] and the conventional group [mean $1.88^{\circ}$ deviated from neutral mechanical alignment (range, varus $4.2^{\circ}$ to valgus $\left.\left.2.8^{\circ}\right), \mathrm{p}=0.043\right]$. One patient $(5 \%)$ in the image-free robotic group and three patients $(15 \%)$ in the conventional group had mechanical axis outliers (error $> \pm 3^{\circ}$ ), but without statistically significant difference between groups ( $p=0.605$ ). (Table 2) (Figure 1) Significant differences were found in the femoral rotation alignment between the image-free robotic group [mean $1.00^{\circ} \pm 0.75^{\circ}$ deviated from the epicondylar axis (range, internal 


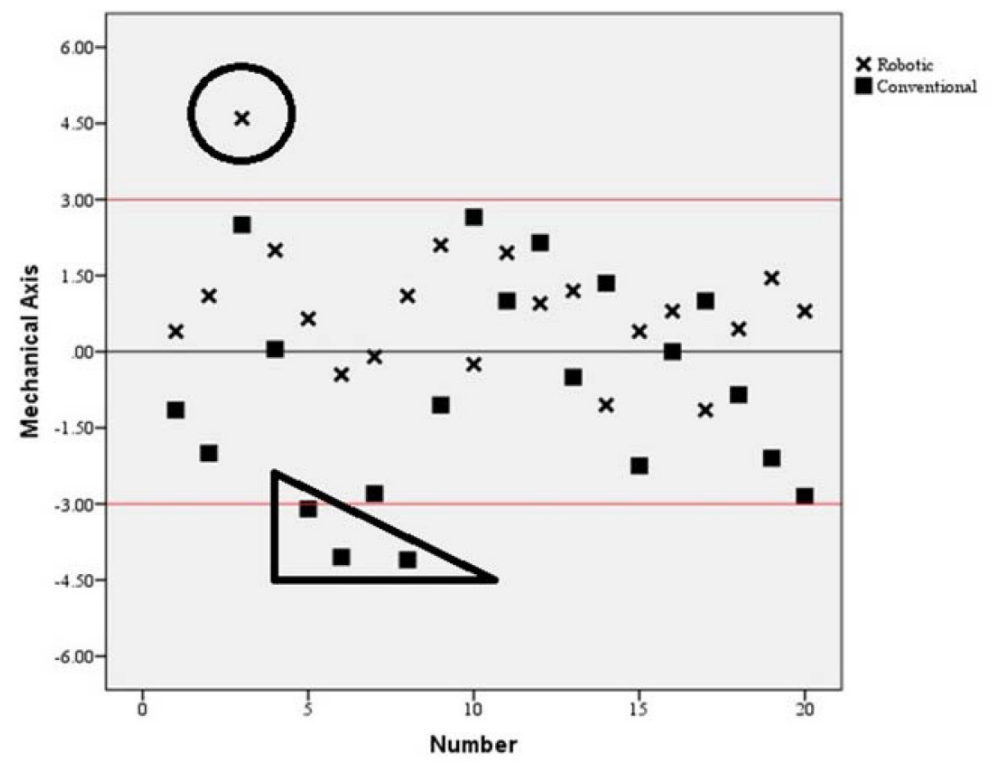

Figure 1. Graphic representation of postoperative mechanical axis comparing between the image-free robotic group and the conventional group (accepted alignment was $\pm 3^{\circ}$ varus/valgus deviation). Negative values stand for varus \& positive values stand for valgus.

rotation $1.6^{\circ}$ to external rotation $\left.3.1^{\circ}\right)$ ] and the conventional group [mean $2.33^{\circ} \pm 0.96^{\circ}$ deviated from the epicondylar axis (range, internal rotation $2.8^{\circ}$ to external rotation $\left.\left.4.9^{\circ}\right), p<0.001\right]$. The mean posterior tibial slope showed no significant differences between both groups $\left(3.89^{\circ} \pm 1.66^{\circ}\right.$ vs. $\left.4.12^{\circ} \pm 1.37^{\circ}, p=0.639\right)$. Significant differences were found in operative time between the image-free robotic and the conventional groups [102.80 $\pm 11.18 \mathrm{~min}$ (range, $81-126 \mathrm{~min}$ ) vs. 62.90 $\pm 3.28 \mathrm{~min}$ (range, 58-69 $\mathrm{min}$ ), respectively, $p<0.001]$. Mean difference time was 39.9 minutes. Mean hemoglobin change in the image-free robotic group was significantly higher than that of the conventional group $[2.24 \pm 0.49 \mathrm{~g} / \mathrm{dl}$ (range, $1.6-3.5 \mathrm{~g} / \mathrm{dl}$ ) vs. $1.64 \pm 0.68 \mathrm{~g} / \mathrm{dl}$ (range, $0.5 \pm 3.3$ $\mathrm{g} / \mathrm{dl}), p=0.001]$. No significant differences were found in length of stay [3.30 days (range, 3-4 days) vs, 3.15 (range, $3-4$ days), $p=0.268$ ] and presenting no postoperative complications such as wound infection, deep vein thrombosis or blood transfusion in both groups.

\section{Discussion}

The goal of primary TKA is to re-establish a normal mechanical axis. Conventional TKA which has been accepted as a standard treatment, performed by manual instrumentation and guided by intramedullary or extramedullary rods, as well as rotational guides that are not patient-specific. Despite continued improvement of manual instrumentation, postoperative component malalignment has still been reported. The mechanical axis of the limb in the coronal plane has been cited more than other alignment parameters. ${ }^{(9,27)}$ Jeffery et al. ${ }^{(28)}$ reported outcomes after TKA among 115 patients with $24 \%$ rate of aseptic loosening when the mechanical axis exceed $\pm 3^{\circ}$ valgus/varus deviation, but a rate of only $3 \%$ for the knee with an axis within $3^{\circ}$ of neutral. The study showed that limb malalignment affected longevity of prosthesis; thus, the mechanical axis within $3^{\circ}$ neutral in the coronal plain remains a reasonable target and should be considered as the standard treatment. Rotational alignment of the femoral component is also important because it can affect patellofemoral tracking, ligament balancing and causes anterior knee pain. Jenny JY, Boeri C et al. ${ }^{(29)}$ reported mal-alignment of the femoral component up to $15^{\circ}$ using conventional techniques. Computer and roboticassisted technology were developed to improve the accuracy of alignment. Several studies had shown better alignment in the robotic group than in the conventional TKA. Song et al. ${ }^{(16)}$ comparing the outcomes of robotic-assisted and conventionalTKAinsamepatient, simultaneously. They found the outliers of postoperative leg alignment of the conventional side more than that of the robotic-assisted side (mechanical axis, coronal inclination of the femoral prosthesis and the sagittal inclination of the tibial prosthesis). 


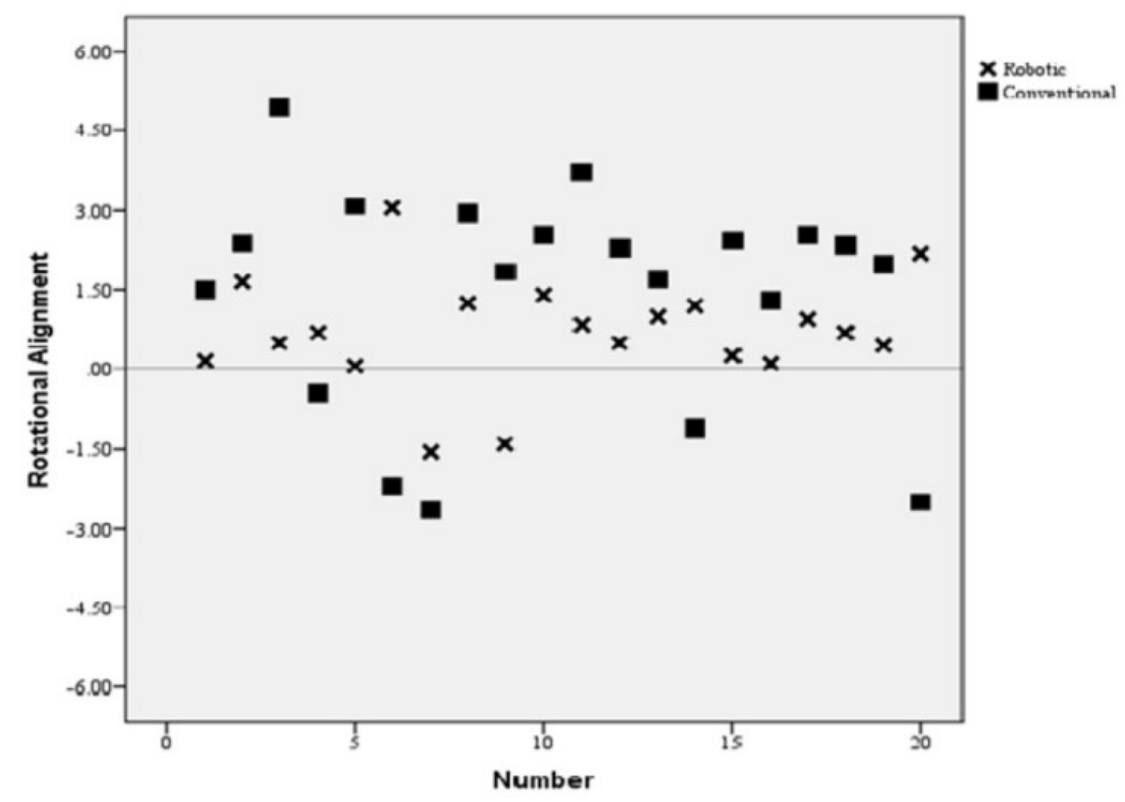

Figure 2. Graphic representation of postoperative rotational alignment comparing between the image-free robotic group and the conventional group. Negative values stand for internal rotation \& positive values stand for external rotation.

Liow et al. ${ }^{(18)}$ reported no mechanical axis outliers $\left(> \pm 3^{\circ}\right.$ from neutral) in 31 patients treated with roboticassisted TKA. The newer technology constitutes the Navio Surgical Systems, a roboticassisted platform using CT-free technology for accurate implant sizing and positioning without the need of rods. While older generation robotic-assisted platforms require pre-operative CT scan, the Navio Surgical System works without them, meaning the patients are not exposed to the potentially harmful radiation.

In this study, we compared the newer technology image-free robotic-assisted TKA (Navio System) with conventional TKA. We found that the mean mechanical alignment and rotational alignment in the image-free robotic group was significantly closer to the mechanical axis and epicondylar axis than that of the conventional group (Figures 1 and 2). The number of mechanical axis outliers was defined as an error of more than $3^{\circ}$ varus or valgus deviation. In the robotic group only one outlier was observed (5\%), and in conventional group three outliers were observed (15\%), without significant difference (Figure 1). These results were similar to one related study showing that the older version robotic assisted TKA exhibited greater accuracy of the component alignment than that of the conventional technique. Despite reducing the component malalignment in the robotic-assisted group, several studies have shown no difference in short term functional outcomes. The recent study of Liow et al. ${ }^{(30)}$ reported a subtle improvement in patient QoL measurement in robotic-assisted TKA compared with conventional TKA at 2 years follow-up.

We observed that surgical wounds were longer in the image-free robotic group because the surgeon required more exposure for tracking fixation. The operative time and blood loss in image-free robotic group were significantly higher than that of the conventional group, probably from time consuming involving registration of the instrument and burring of bone. However, operative time and blood loss in the image-free robotic group decreased in the later case because the surgeon had gained more experience using the Navio Surgical System. Despite these issues, data demonstrated significant improvement of alignment, current disadvantages such as the need for the placement of tracking fixation, increased operating time, increased blood loss and higher overall cost that will have to be resolved in the future. Further randomized and multicenter randomized controlled trials might be required to evaluate the accuracy and clinical efficacy to support this technology. Cost effectiveness will need a long term study in which improved in component alignment could save operative cost or reduce late failure of prosthesis. 


\section{Conclusion}

Image-free robotic-assisted TKA is a surgical procedure that provides better accuracy in prosthetic alignment in both mechanical axis and rotational axis compared with conventional TKA. However, the image-free robotic-assisted TKA had some disadvantages such as more blood loss, longer operative time and requiring more experience than conventional TKA

\section{References}

1. Cates HE, Ritter MA, Keating EM, Faris PM. Intra-medullary femoral alignment systems in total knee replacement. Colin Orthop Relat Res 1993; 286: 32-9.

2. Peterson TL, Engh GA. Radiographic assessment of knee alignment after total knee arthroplasty. J Arthroplasty 1988; 3: 67-72.

3. Simmons ED Jr, Sullivan JA, Rackemann S, Scott RD. The accuracy of tibial intramedullary alignment devices in total knee arthroplasty. J Arthroplasty 1991; 6: 45-50.

4. Dennis DA, Channer M, Susan MH, Stringer EA. Intramedullary versus extramedullary tibial alignment systems in total knee arthroplasty. J Arthroplasty 1993; 8: 43-7.

5. Furnes O, Espehaug B, Lie SA, Vollset SE, Engesaeter LB, Havelin LI. Failure mechanisms after unicompartmental and tricompartmental primary knee replacement with cement. J Bone Joint Surg 2007; 89: 519-25.

6. Scuderi GR, Insall JN, Windsor RE, Moran MC. Survivorship of cemented knee replacements. J Bone Joint Surg Br 1989; 71: 798-803.

7. Emmerson KP, Moran CG, Pinder IM. Survivorship analysis of the Kinematic Stabilizer total knee replacement: a 10-to 14-year follow-up. J Bone Joint Surg Br 1996; 78-B: 441-5.

8. Ranawat CS, Flynn WF, Saddler S, Hansraj KK, Maynard MJ. Long-term results of the total condylar knee arthroplasty. A 15-year survivorship study. Clin Orthop Relat Res 1993; 286: 94-102.

9. Lotke PA, Ecker ML. Influence of positioning of prosthesis in total knee replacement. J Bone Joint Surg 1977; 59: 77-9.

10. Moreland JR. Mechanisms of failure in total knee arthroplasty. Clin Orthop Relat Res 1988; 266: 49-64.
11. Boyd AD, Ewald FC, Thomas WH. Long-term complications after total knee arthroplasty with or without resurfacing of the patella. $J$ Bone Joint Surg Am 1993; 75: 674.

12. Laskin RS. Flexion space configuration in total knee arthroplasty. J Arthroplasty 1995; 10: 657-60.

13. Barrack RL, Schrader T, BertotAJ, Wolfe MW, Myers L. Component rotation and anterior knee pain after total knee arthroplasty. Clin Orthop Relat Res 2001; 392: 46-55.

14. Bellemans J, Vandenneucker H, Vanlauwe J. Robot-assisted total knee arthroplasty. Clin Orthop Relat Res 2007; 464: 111-6.

15. Liow MH, Chin PL, Tay KJ, Chia SL, Lo NN, Yeo SJ. Early experiences with robot-assisted total knee arthroplasty using the DigiMatch ROBODOC(R) surgical system. Singap Med J 2014; 55: 529-34.

16. Song EK, Seon JK, Park SJ, Jung WB, Park HW, Lee GW. Simultaneous bilateral total knee arthroplasty with robotic and conventional techniques: a prospective, randomized study. Knee Surg Sports Traumatol Arthrosc 2011; 19: 1069-76.

17. Decking J, Theis C, Achenbach T, Roth E, Nafe B, Eckardt A. Robotic total knee arthroplasty: the accuracy of CT-based component placement. Acta Orthop Scand 2004; 75: 573-9.

18. Liow MH, Xia Z, Wong MK, Tay KJ, Yeo SJ, ChinPL.Robot-assisted totalkneearthroplasty accurately restores the joint line and mechanical axis. A prospective randomised study. J Arthroplasty 2014; 29: 2373-7.

19. Siebert W, Mai S, Kober R, Heeckt PF. Technique and first clinical results of robot-assisted total knee replacement. Knee 2002; 9: 173-80.

20. Song EK, Seon JK, Yim JH, Netravali NA, Bargar WL. Robotic-assisted TKA reduces postoperative alignment outliers and improves gap balance compared to conventional TKA. Clin Orthop Relat Res 2013; 471: 118-26.

21. Coon TM. Integrating robotic technology into the operating room. Am J Orthop (Belle Mead NJ) 2009; 38(2 Suppl): 7-9.

22. Park SE, Lee CT. Comparison of roboticassisted and conventional manual implantation of a primary total knee arthroplasty. J Arthroplasty 2007; 22: 1054-9. 
23. Lonner JH, Smith JR, Picard F, Hamlin B, Rowe PJ, Riches PE. High degree of accuracy of a novel image-free handheld robot for unicondylar knee arthroplasty in a cadaveric study. Clin Orthop 2015; 473: 206-12.

24. Barrack RL, Schrader T, Bertot AJ, Wolfe MW, Myers L. Component rotation and anterior knee pain after total knee arthroplasty. Clin Orthop Relat Res 2001; 392: 46-55.

25. Berger RA, Crossett LS, Jacobs JJ, Rubash HE. Malrotation causing patellofemoral complications after total knee arthroplasty. Clin Orthop Relat Res 1998; 356: 144-53.

26. Berger RA, Rubash HE, Seel MJ, Warren HT, Crosset LS. Determining the rotational alignment of the femoral component in total knee arthroplasty using the epicondylar axis. Clin Orthop Relat Res 1993; 286: 40-7.
27. Vince KG, Insall JN, Kelly MA. Total condylar prosthesis: 10- to 12-year results of a cemented knee replacement. J Bone Joint Surg Br 1989; 71-B: 793-7.

28. Jeffery RS, Morris R, Denham RA. Coronal alignment after total knee replacement. J Bone Joint Surg Br 1991; 73-B: 709-14.

29. Jenny JY, Boeri C. Low reproducibility of the intra-operative measurement of the transepicondylar axis during total knee replacement. Acta Orthop Scand 2004: 75: 74-7.

30. Liow MHL, Goh GS, Wong MK, Chin PL, Tay DK, Yeo SJ. Robotic assisted total knee arthroplasty may lead to improvement in quality of life measures: a 2 year follow up of a prospective randomized trial. Knee Surg Sports Traumatol Arthrosc 2017; 9: 2942-51. 